\title{
Microfluidic flow direction and rate vector sensor based on a partially gold-coated TFBG
}

\author{
Changyu Shen \\ China Jiliang University \\ Dejun Liu \\ Shenzhen University \\ Xiaokang Lian \\ Technological University Dublin
}

See next page for additional authors

Follow this and additional works at: https://arrow.tudublin.ie/prcart

Part of the Electrical and Computer Engineering Commons, and the Optics Commons

\section{Recommended Citation}

Changyu Shen, Dejun Liu, Xiaokang Lian, Tingting Lang, Chunliu Zhao, Yuliya Semenova, and Jacques Albert, "Microfluidic flow direction and rate vector sensor based on a partially gold-coated TFBG," Opt. Lett. 45, 2776-2779 (2020)

This Article is brought to you for free and open access by the Photonics Research Centre at ARROW@TU Dublin. It has been accepted for inclusion in Articles by an authorized administrator of ARROW@TU Dublin. For more information, please contact arrow.admin@tudublin.ie, aisling.coyne@tudublin.ie,gerard.connolly@tudublin.ie. Funder: National Natural Science Foundation of China

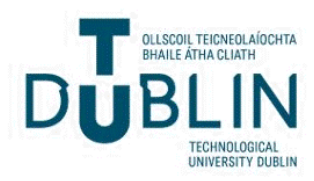




\section{Authors}

Changyu Shen, Dejun Liu, Xiaokang Lian, Tingting Lang, Chunliu Zhao, Yuliya Semenova, and Jacques Albert 


\title{
Microfluidic flow direction and rate vector sensor based on a partially gold-coated TFBG
}

\author{
Changyu Shen, ${ }^{1, *}$ (1) Dejun Liu, ${ }^{2}$ (i) Xiaokang Lian,,${ }^{4}$ Tingting Lang, ${ }^{1}$ Chunliu Zhao, ${ }^{1}$ \\ Yuliya Semenova, ${ }^{4}$ and Jacques Albert ${ }^{3}$ (i) \\ ${ }^{1}$ Institute of Optoelectronic Technology, China Jiliang University, Hangzhou 310018, China \\ ${ }^{2}$ College of Physics and Optoelectronic Engineering, Shenzhen University, Shenzhen 518060, China \\ ${ }^{3}$ Department of Electronics, Carleton University, Ottawa, Ontario K1S 5B6, Canada \\ ${ }^{4}$ Photonics Research Center, Dublin Institute of Technology, Kevin Street, Dublin 8, Ireland \\ *Corresponding author: shenchangyu56@163.com
}

Received 13 March 2020; revised 3 April 2020; accepted 8 April 2020; posted 8 April 2020 (Doc. ID 392511); published 12 May 2020

In microfluidic chips applications, the monitoring of the rate and the direction of a microfluidic flow is very important. Here, we demonstrate a liquid flow rate and a direction sensor using a partially gold-coated tilted fiber Bragg grating (TFBG) as the sensing element. Wavelength shifts and amplitude changes of the TFBG transmission resonances in the near infrared reveal the direction of the liquid flowing along the fiber axis in the vicinity of the TFBG due to a nanoscale gold layer over part of the TFBG. For a device length of $10 \mathrm{~mm}$ (and a diameter of $125 \mu \mathrm{m}$ for easy insertion into microfluidic channels), the flow rates and the direction can be detectable unequivocally. The TFBG waveguiding properties allow such devices to function in liquids with refractive indices ranging from 1.33 to about 1.40. In addition, the proposed sensor can be made inherently temperature-insensitive by referencing all wavelengths to the wavelength of the core mode resonance of the grating, which is isolated from the fiber surroundings. () 2020 Optical Society of America

https://doi.org/10.1364/OL.392511

The microfluidic flow rate and the direction are important to microfluidic technology applications. For example, the flow rate dominates cell adhesion and monoclonal antibody production in biopharmaceutical testing [1] in the size and the generation speed of the droplets in flow-focusing generators [2] and in the speed and efficiency of counting and sorting in flow cytometry [3]. Several methods have been demonstrated for flow rate detection, such as the electrical admittance [4] or cantilever deflection [5] based micro electro-mechanical systems (MEMS), optical fiber flowmeters based on laser Doppler velocimetry [6], fiber Bragg gratings (FBGs) [7-9], optical fiber cantilevers [10-13], fiber "hot-wires" [14-16], or Fabry-Perot interferometers [17]. However, the MEMS flow rate sensors involve complex fabrication processes and materials or processes that may not be suitable for exposure to certain liquids of interest for biological and chemical laboratories (conductive ionic liquids will short electrodes, for instance). For the optical fiber flowmeters, most of them can only detect the flow rate. Other methods, such as an FBG cantilever and a silicon Fabry-Perot interferometers-based flow direction sensor, suffer from the drawbacks of large volume, low sensitivity, and crosssensitivity to temperature. And so far, no perfect fiber sensor can measure the microfluidic liquid flow rate and the direction simultaneously.

In this Letter, we demonstrate a novel optical fiber sensor for the detection of the microfluidic flow rate and the direction, which address the limitations described above. The device is derived from a recently developed flow rate sensor based on a tilted FBG (TFBG) [18], but with an important improvement. By coating part of the TFBG with a nanoscale gold coating, the measured transmission spectrum of the device depends not only on flow rate but also on which end of the device is first immersed in the fluid or exposed to a fluid change. Other combinations of TFBGs or coatings would also serve the same purpose, but the nanoscale gold layer has the advantage of accelerating the changes in the transmission spectrum with a negligibly thin diameter increase due to plasmonic effects [19]. As in most devices based on TFBGs, light power and temperature fluctuations can be referenced out of any spectral measurement by measuring the position and amplitude of the transmission resonance of the single core mode, which is by definition insensitive to changes in the fluid surrounding the fiber [19-24]. This sensor can detect the flow rate at the same time, and more important, the flow direction can be obtained.

Figure 1 illustrates the four possible waveguiding situations for light propagating through a partially gold-coated TFBG: (a) totally in air, (b) fully immersed in water, (c) only part I of the TFBG (gold-coated side) in water, or (d) only part II of the TFBG (uncoated side) in water. Each of these configurations results in a different transmission spectrum for light launched in the core of the fiber, and as shown earlier, it is possible to extract the rate at which liquids infiltrate each section with a single, time-controlled sweep of a spectral interrogation system [18]. For the experiments to be described here, a TFBG with a total length of $10 \mathrm{~mm}$ and a grating period of $542 \mathrm{~nm}$ was inscribed in the core of a single mode, hydrogen-loaded optical fiber with an argon ion laser and the phase mask technique [19]. The grating fringes were inclined by 8 deg relative to the fiber axis to 

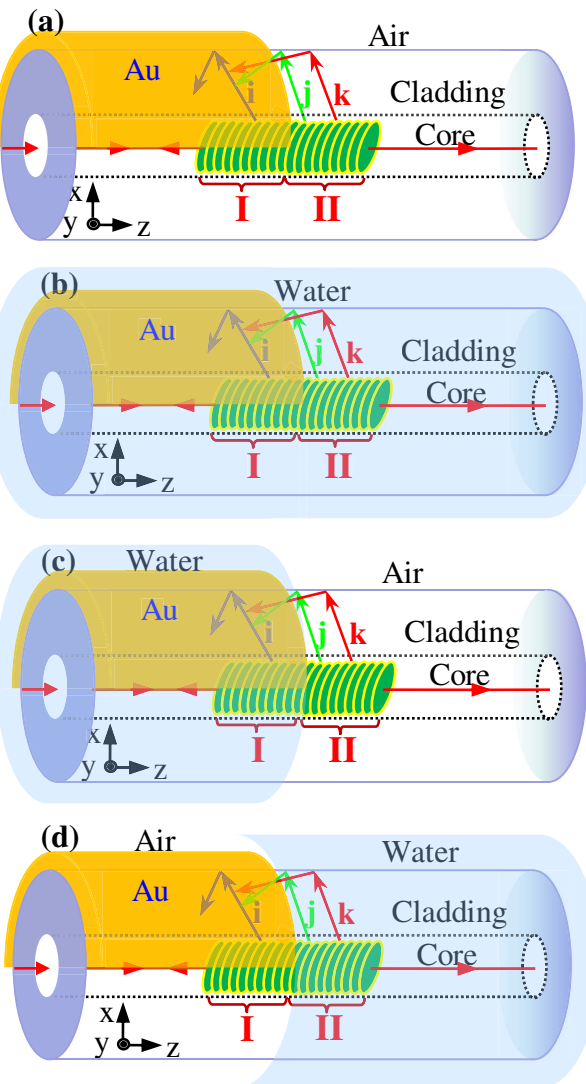

Fig. 1. Schematic diagrams of the partially gold-coated TFBG: (a) totally in air, (b) fully immersed in water, (c) only part I (gold-coated side) in water, (d) only part II (uncoated side) in water.

increase the coupling efficiency between the single mode guided by the core and a large number of cladding guided light modes. The evanescent field of these cladding modes extends outside the cladding surface, and when changes in the surrounding media occur, the amplitudes and the wavelengths of the mode resonances are modified measurably $[19,20]$. In order to provide directional sensitivity, a $50 \mathrm{~nm}$ thick gold layer was deposited on the surface of the fiber cladding over half the length of the TFBG by electron beam evaporation (Temescal, FC-2000). While it is customary to aim for uniform coatings on fiber sensor devices, and gold-coated fiber surface plasmon devices in particular, by rotating the fiber in the deposition chamber, in this particular case, the fiber was fixed and only half the circumference was covered in gold. A sharp boundary between the bare and goldcoated sections was obtained by shielding half the TFBG by a Perspex (acrylic) tube (positioned with a microscope over the marked TFBG position) during the deposition.

For each of the two sections of the TFBG, the transmission spectrum resonances are governed by mode coupling equations, and in particular by the standard phase-matching condition for the resonance wavelength of the core mode $\lambda_{\text {Bragg }}$ and of any $i$ th cladding mode $\lambda_{\text {clad }}^{i}$ in the TFBG transmission spectrum [19]:

$$
\begin{gathered}
\lambda_{\text {Bragg }}=2 n_{\text {eff }}(\text { core }) * \Lambda / \cos (\theta), \\
\lambda_{\text {clad }}^{i}=\left(n_{\text {eff }}(\text { core })+n_{\text {eff }}^{i}(\text { clad })\right) * \Lambda / \cos (\theta),
\end{gathered}
$$

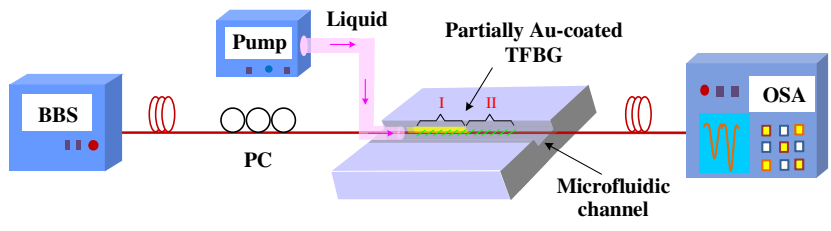

Fig. 2. Schematic diagram of the experimental setup.

where $n_{\text {eff }}$ (core) and $n_{\text {eff }}^{i}$ (clad) are the effective indices of the fiber core and the cladding modes, respectively. $\Lambda$ is the grating period normal to the grating planes, and $\theta$ is the tilt angle of the grating planes relative to the transverse plane of the fiber. The length of the gold coating along the fiber axial direction on the TFBG surface was controlled strictly to $5 \mathrm{~mm}$, i.e., half the total grating length. Accurate control of the distribution and thickness of the gold around the circumference of the fiber by the single-side deposition is more difficult to evaluate, but it is not an important factor, since the function of the gold coating is to produce effective refractive index differences between the cladding modes of the two half-lengths of the grating [parts I and II, as shown in Fig. 1(a)]. According to Eqs. (1) and (2), as the surrounding medium of the TFBG medium changes, $n_{\text {eff }}$ (core) remains constant, and so does $\lambda_{\text {Bragg }}$. However, for the four cases in Figs. $1(\mathrm{a})-1(\mathrm{~d})$, the $n_{\text {eff }}^{i}$ (clad) are different, as they depend on both the presence (or absence) of the coating and the nature of the external medium. Therefore, as the water flows from part I to part II of the TFBG [as illustrated in Fig. 1(c)], or in the opposite direction, from part II to part I [as shown in Fig. 1(d)], the effective indices experienced by the cladding modes change, which results in different shifts of the cladding mode resonances, while the core mode resonance remains un-perturbed and thus can be used both for temperature compensation and optical power referencing [19].

Figure 2 shows the schematic diagram of the experimental setup, with water as a test liquid. A fiber-coupled Broadband Source (BBS) (THORLABS) with a wavelength range from $1450 \mathrm{~nm}$ to $1650 \mathrm{~nm}$ was used as the light source. An optical spectrum analyzer (OSA) (Agilent, 86142B) with a resolution of $0.05 \mathrm{~nm}$ was used to record the transmission spectra for S-polarized input light and P-polarized input light. Linearly polarized light is obtained from a polarization controller (PC) in order to select only one of the two groups of vector-polarized cladding modes excited by the TFBG (due to the inclination of the grating planes, which breaks the cylindrical symmetry of the fiber) because the response of the cladding modes to metal coatings depends strongly on the polarization of the mode evanescent fields. Here, S-polarized light (relative to the plane of inclination of the grating fringes) was used, thus selecting the $\mathrm{TE}_{0 n}$ and $\mathrm{HE}_{m n}$ cladding modes only (with $m$ being the azimuthal mode index and $n$ being the radial index). For the detection of the water ingress rate and the direction in the microfluidic chip application, the partially gold-coated TFBG was fixed to lie along the middle of the microfluidic channel. The water was pumped from the reservoir through a calibrated micro-flow pump (YZ1515, Baoding Qili constant Pump, Co.). For the calibration of the four cases shown in Fig. 1, the position of the partially gold-coated TFBG and of the coated section were marked on the microfluidic channel so that the representative power spectra could be measured statically with water inserted from either end and not moving. 


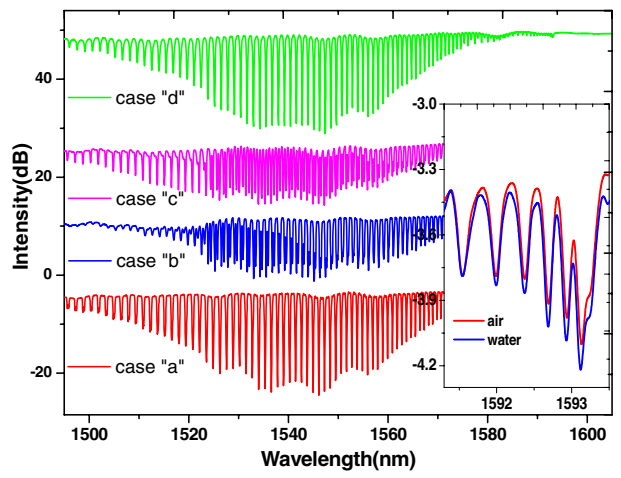

Fig. 3. Measured transmission spectra of the partially gold-coated TFBG in air (case "a" from Fig. 1), completely covered by water (case "b"), and with either only the gold-coated section in water (case "c") or only the bare section in water (case "d"). Inset: Closeup on the low order mode resonances.

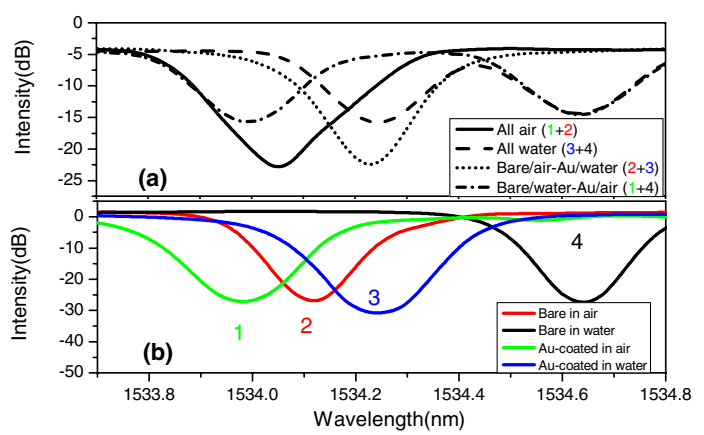

Fig. 4. (a) Spectra of the partially gold-coated TFBG fully in air and water, as well as with either the bare or coated section in water. (b) Spectra of fully coated and bare TFBGs in air and water (see text for details).

Figure 3 shows the transmission spectra of the partially gold-coated TFBG for the four cases corresponding to Figs. 1(a) -1 (d). On the scale of Fig. 3, the different cases are clearly distinguishable but quite complex. The inset of Fig. 3 shows that the low-order cladding modes at longer wavelengths (these modes are well confined in the cladding) are indeed invariant and can be used as a wavelength and a power reference to measure spectra on a finer scale.

Clear differences between the different cases become evident in the spectral region shown in Fig. 4(a). These differences can be elucidated by observing the equivalent resonance for $10 \mathrm{~mm}$ long bare and fully coated TFBGs in air and water, as shown in Fig. 4(b).

It turns out that when the half-coated grating is entirely in air (case "a," Fig. 1), the spectral response is a superposition of the resonances labeled 1 and 2 in Fig. 4(b); but since each half grating is only $5 \mathrm{~mm}$ long, the resonances widen and overlap. The same occurs for the overlap of resonances 2 and 3 for the case of the bare half in air and the coated half in water (case "c," Fig. 1). For the bare half in water and the coated half in air (case "d," Fig. 1), the individual resonances (1 and 4) are sufficiently far apart and the spectrum shows two individual resonances. Finally, for the two halves both in water (case "b," Fig. 1), it is resonances 3 and 4 that make up the spectrum, and they are also
Table 1. Combination of Uniform Grating Spectra (Gold Coated or Bare, in Air or in Water)

\begin{tabular}{lccc}
\hline & $\begin{array}{c}\text { Uniform } \\
\text { Grating } \\
\text { Spectrum }\end{array}$ & $\begin{array}{c}\text { Partially } \\
\text { Coated } \\
\text { Grating Case }\end{array}$ & $\begin{array}{c}\text { Combination of } \\
\text { Uniform } \\
\text { Grating Spectra }\end{array}$ \\
\hline $\begin{array}{l}\text { Coated TFBG in } \\
\text { air }\end{array}$ & 1 & a: all in air & $1+2$ \\
$\begin{array}{l}\text { Bare TFBG in air } \\
\text { Coated TFBG in }\end{array}$ & 2 & b: all in water & $3+4$ \\
$\begin{array}{l}\text { water } \\
\text { Bare TFBG in }\end{array}$ & 4 & $\begin{array}{c}\text { c: gold part in } \\
\text { water }\end{array}$ & $2+3$ \\
water & 4 & $\begin{array}{c}\text { d: bare part in } \\
\text { water }\end{array}$ & $1+4$ \\
\end{tabular}
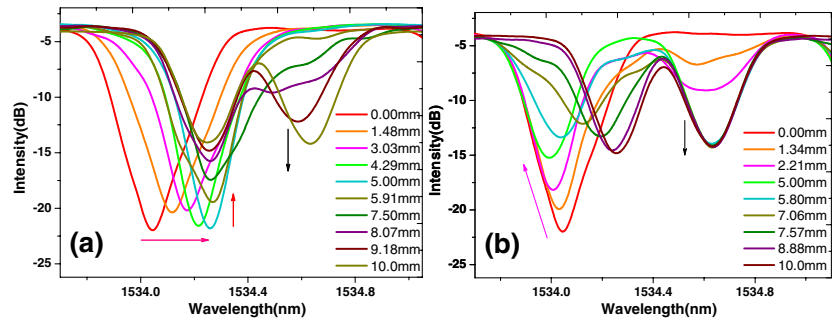

Fig. 5. Spectral changes with the water flowing through the partially gold-coated TFBG along the fiber axis: (a) from the gold-coated end to the uncoated end; (b) from the uncoated end to the gold-coated end.

separated enough to show up individually in Fig. 4(a). Table 1 summarizes these four situations, and it is understood that when water progresses from one end to the other, the spectral response will evolve smoothly in sequence case "a"-case "c"-case "b" (if the flow is from the coated side toward the bare side), and in sequence case "a"-case "b"-case " $d$ " for the other direction of flow. Of course, if the initial condition is fully immersed, the removal of the liquid can also be followed in similar fashion.

For the dynamic case, i.e., water gradually covering the device from one end to the other, the spectral shifts are continuous as shown in Fig. 5. Figure 5(a) shows the changes in the transmission spectrum as water flows gradually along the TFBG from the gold-coated end toward its uncoated end. The legend shows the corresponding lengths of the TFBG covered by water. It can be seen that the wavelength corresponding to case "a" (1534.05 nm) gradually red shifts to that of case "c" $(1534.26 \mathrm{~nm})$ as the contribution from the gold-coated section moves from its air value to its water value when the watercovered length of the TFBG increases from 0 to $5 \mathrm{~mm}$. Beyond the $5 \mathrm{~mm}$ length, as the water continues to flow, the resonant wavelength no longer shifts, but its intensity decreases as water gradually covers the remaining bare section, whose resonance shifts from its air value to its water coated value at $1534.65 \mathrm{~nm}$. If the water flows in the opposite direction, (i.e. case "a" to "d" and then to "b"), Figure 5(b) shows that the initial resonant wavelength at $1534.05 \mathrm{~nm}$ gradually blue shifts to $1533.99 \mathrm{~nm}$, because part of the bare section contribution now contributes to its resonance value in water. This is followed by the gradual transfer of the resonance of the gold-coated section from its value in air to its value in water.

Now in order to obtain quantitative information about the direction of the flow and the feasibility for the flow rate measurements, Fig. 6 shows the evolution of the amplitude and the 

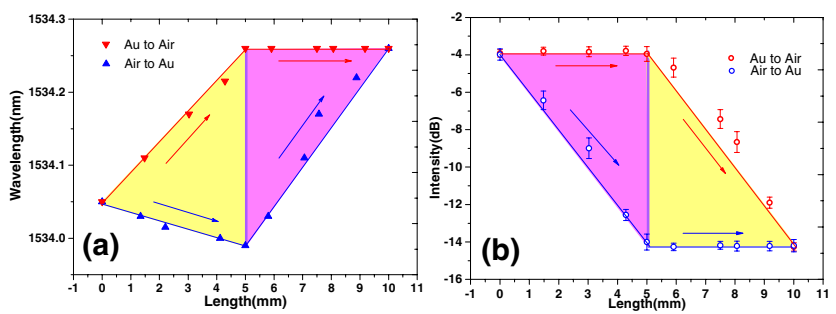

Fig. 6. Evolution of: (a) the wavelength of the $1534.05 \mathrm{~nm}$ resonance and (b) the amplitude of the $1534.65 \mathrm{~nm}$ resonance, during immersion from the coated end (in red) and from the uncoated end (in blue).

wavelength of the dominant resonances at $1534.05 \mathrm{~nm}$ and $1534.65 \mathrm{~nm}$ during immersion from the coated end and the uncoated end as extracted from the spectra of Fig. 5. In Fig. 6(a), the wavelength changes of the $1534.05 \mathrm{~nm}$ resonance are plotted as a function of immersion length for the two directions of the flow. For Au to air, the wavelength changes rapidly over the first $5 \mathrm{~mm}$ and then remains fixed for the remainder of the flow across the TFBG. In the other direction, a relatively small blue shift over the first half is followed by a large red shift for the last $5 \mathrm{~mm}$. In order to allow high sensitivity measurements over the full length in both directions, Fig. 6(b) shows that high rate intensity changes of the resonance at $1534.65 \mathrm{~nm}$ occur for the situations where the wavelength change rates are small or zero in Fig. 6(a), thereby enabling accurate water-air boundary measurements along the full length, and for both directions.

It is obvious from Fig. 6 that by monitoring both the wavelength near $1534.05 \mathrm{~nm}$ and the intensity of the $1534.65 \mathrm{~nm}$ resonance, the location of the water boundary along the grating can be determined unequivocally. The rates of change are approximately $40 \mathrm{pm} / \mathrm{mm}$ and $2 \mathrm{~dB} / \mathrm{mm}$ for the wavelength and the intensity, respectively, and therefore repeated spectral measurements across 1534-1534.7 nm can be used to find the rate of infiltration of water for both directions. With a typical fiber grating interrogator, the grating resonance features can be determined with $10 \mathrm{pm}$ accuracy routinely at rates from $1 \mathrm{~Hz}$ to at least $1 \mathrm{kHz}$, meaning that the flow rates that can be measured are at least from 0 to $100 \mathrm{~mm} / \mathrm{s}$ (accounting for some data processing time between spectral sweeps). Note here that increasing the length would narrow the resonances (seen in Fig. 4) and make them easier to follow accurately if the coupling strength would be reduced at the same time. Increasing the length would also decrease the slopes (rate of change with length) in Fig. 6, and thus may increase the accuracy of the position determination. Decreasing the length would have the opposite effects: higher slopes in Fig. 6 and broader resonances in Fig. 4, therefore undesirable. This partially gold-coated TFBG based sensor can also be used for other liquids (with RIs ranging from 1.33 to 1.40 , with adjustment of the wavelengths involved) flow direction and the rate measurement, since the behavior of the cladding modes and the resonant wavelength characteristics are similar. In addition, the Bragg wavelength can be used for self-compensation to account for the variations in the external temperature. Just as always in high-accuracy TFBG spectral measurements, in order to compensate for the potential temperature difference between the measurements, the spectra should be shifted along the wavelength axis to make their Bragg wavelengths coincide.
In conclusion, a simple, partially gold-coated TFBG based optical fiber device for determining the liquid flow direction in microfluidics over multi-mm distances and the feasibility for flow rate sensing up to $100 \mathrm{~mm} / \mathrm{s}$ are presented. The water flow direction and rate can be detected through continuous spectral measurements of the wavelength and intensity of two cladding mode resonances as a function of time, only requiring a conventional FBG interrogator with at least $10 \mathrm{pm}$ wavelength accuracy and $1 \mathrm{kHz}$ spectrum acquisition rate. In addition, due to the TFBG's internal temperature compensation mechanism, any external surrounding temperature induced wavelength shift can be eliminated by its Bragg wavelength's calibration.

Funding. National Natural Science Foundation of China (11874332, 61727816, 61775202, 61875251).

Acknowledgment. The authors would like to thank Prof. Tuan Guo (Jinan University, China) for his helpful comments.

Disclosures. The authors declare no conflicts of interest.

\section{REFERENCES}

1. L. D. Garza-García, E. García-López, S. Camacho-León, M. D. R. Rocha-Pizaña, F. López-Pacheco, J. López-Meza, D. Araiz-Hernández, E. J. Tapia-Mejía, G. T. Santiago, C. A. Rodríguez-González, and M. M. Alvarez, Lab Chip 14, 1320 (2014).

2. C. A. Stan, S. K. Y. Tang, and G. M. Whitesides, Anal. Chem. 81, 2399 (2009).

3. U. Hassan, N. N. Watkins, C. Edwards, and R. Bashir, Lab Chip. 14, 1469 (2014).

4. G. J. M. Krijnen, M. Dijkstra, J. J. Baar, S. S. Shankar, W. J. Kuipers, R. J. H. Boer, D. Altpeter, T. S. J. Lammerink, and R. Wiegerink, Nanotechnology 17, S84 (2006).

5. A. Quist, A. Chand, S. Ramachandran, D. Cohen, and R. La, Lab Chip 6, 1450 (2006).

6. W. N. MacPherson, J. D. C. Jones, B. J. Mangan, J. C. Knight, and P. St. J. Russell, Opt. Commun. 223, 375 (2003).

7. S. Takashima, H. Asanuma, and H. Niitsuma, Sens. Actuators A 116, 66 (2004)

8. Y. Zhao, K. Chen, and J. Yang, Measurement 38, 230 (2005).

9. P. Lu and Q. Chen, Meas. Sci. Technol. 19, 125302 (2008).

10. L. Yuan, J. Yang, and Z. Liu, IEEE Sens. J. 8, 1114 (2008).

11. V. Lien and F. Vollmer, Lab Chip 7, 1352 (2007).

12. M. S. Cheri, H. Latifi, J. Sadeghi, M. S. Moghaddam, H. Shahraki, and H. Hajghassem, Analyst 139, 431 (2014).

13. A. S. Nezhad, M. Ghanbari, C. G. Agudelo, M. Packirisamy, R. B. Bhat, and A. Geitmann, IEEE Sens. J. 13, 601 (2013).

14. Z. Liu, M. L. V. Tse, A. P. Zhang, and H. Y. Tam, Opt. Lett. 39, 5877 (2014).

15. R. Chen, A. Yan, Q. Wang, and K. P. Chen, Opt. Lett. 39, 3966 (2014).

16. Y. Li, G. Yan, L. Zhang, and S. He, Opt. Express 23, 9483 (2015).

17. G. Liu, Q. Sheng, W. Hou, and M. Han, Opt. Lett. 41, 4629 (2016).

18. C. Shen, X. Lian, V. Kavungal, C. Zhong, D. Liu, Y. Semenova, G. Farrell, J. Albert, and J. F. Donegan, Opt. Lett. 43, 751 (2018).

19. J. Albert, L. Y. Shao, and C. Caucheteur, Laser Photon. Rev. 7, 83 (2013).

20. T. Guo, F. Liu, B. O. Guan, and J. Albert, Opt. Laser Technol. 78, 19 (2016).

21. W. Zhou, Y. Zhou, and J. Albert, Laser Photon. Rev. 11, 1600157 (2017).

22. W. Zhou, D. J. Mandia, S. T. Barry, and J. Albert, Opt. Lett. 40, 1713 (2015).

23. C. Shen, Y. Zhang, W. Zhou, and J. Albert, Appl. Phys. Lett. 104, 071106 (2014).

24. Y. Wang, C. Shen, W. Lou, F. Shentu, C. Zhong, X. Dong, and L. Tong, Appl. Phys. Lett. 109, 031107 (2016). 